\title{
Universality of Boundary Charge Fluctuations
}

\author{
Clara S. Weber®, ${ }^{1}$ Kiryl Piasotski®, ${ }^{1}$ Mikhail Pletyukhov $\odot,{ }^{1}$ Jelena Klinovaja, ${ }^{2}$ Daniel Loss $\odot,{ }^{2}$ \\ Herbert Schoeller, ${ }^{1}$ and Dante M. Kennes $\odot^{1,3,{ }^{*}}$ \\ ${ }^{1}$ Institut für Theorie der Statistischen Physik, RWTH Aachen, 52056 Aachen, Germany and Jülich Aachen Research Alliance (JARA), \\ Fundamentals of Future Information Technology, Jülich 52428, Germany \\ ${ }^{2}$ Department of Physics, University of Basel, Klingelbergstrasse 82, CH-4056 Basel, Switzerland \\ ${ }^{3}$ Max Planck Institute for the Structure and Dynamics of Matter, Center for Free Electron Laser Science, \\ 22761 Hamburg, Germany
}

(Received 19 August 2020; accepted 24 November 2020; published 8 January 2021)

\begin{abstract}
We establish the quantum fluctuations $\Delta Q_{B}^{2}$ of the charge $Q_{B}$ accumulated at the boundary of an insulator as an integral tool to characterize phase transitions where a direct gap closes (and reopens), typically occurring for insulators with topological properties. The power of this characterization lies in its capability to treat different kinds of insulators on equal footing, being applicable to transitions between topological and nontopological band, Anderson, and Mott insulators alike. In the vicinity of the phase transition, we find a universal scaling $\Delta Q_{B}^{2}\left(E_{g}\right)$ as a function of the gap size $E_{g}$ and determine its generic form in various dimensions. For prototypical phase transitions with a massive Dirac-like bulk spectrum, we demonstrate a scaling with the inverse gap in one dimension and a logarithmic one in two dimensions.
\end{abstract}

DOI: 10.1103/PhysRevLett.126.016803

Introduction. - In the last few decades, studies concerning topological phases of matter, i.e., phases not characterized by a Landau-type of order parameter, have moved to the vanguard of condensed matter research [1-12]. A topological phase transition separates two insulating phases with different topological properties and is typically accompanied by a band inversion at a special point in quasimomentum space, where two bands are directly coupled. Whereas standard metal-insulator transitions are described via localization theories [13,14], a topological phase transition probes specific low-energy features and is characterized by a closing and reopening of a direct gap, accompanied by a change of a topological index. Independent of whether a topological index remains the same or not at such a transition, the fundamental question arises on how to embed these special phase transitions into conventional ones, where the fluctuations of an appropriate observable diverge at the transition, accompanied by the divergence of a characteristic length scale. Close to the transition, such a diverging length scale is naturally given by $\xi_{g}=v_{F} / E_{g}$, where $v_{F}$ is a typical velocity and $E_{g}$ denotes the gap size. Going one step further, this poses the interesting issue of whether fluctuations reveal universal scaling laws as a function of $\xi_{g}$ (or, equivalently, $E_{g}$ ).

Recently it has been proposed that the boundary charge $Q_{B}$ accumulated at a $D-1$-dimensional flat surface of a $D$-dimensional insulator probes universal properties of topological insulators at low energies [15-20]. Close to the transition point, it was demonstrated for onedimensional, single-channel models that $Q_{B}$ directly probes the phase of the gap parameter (in units of $2 \pi$ ) independent

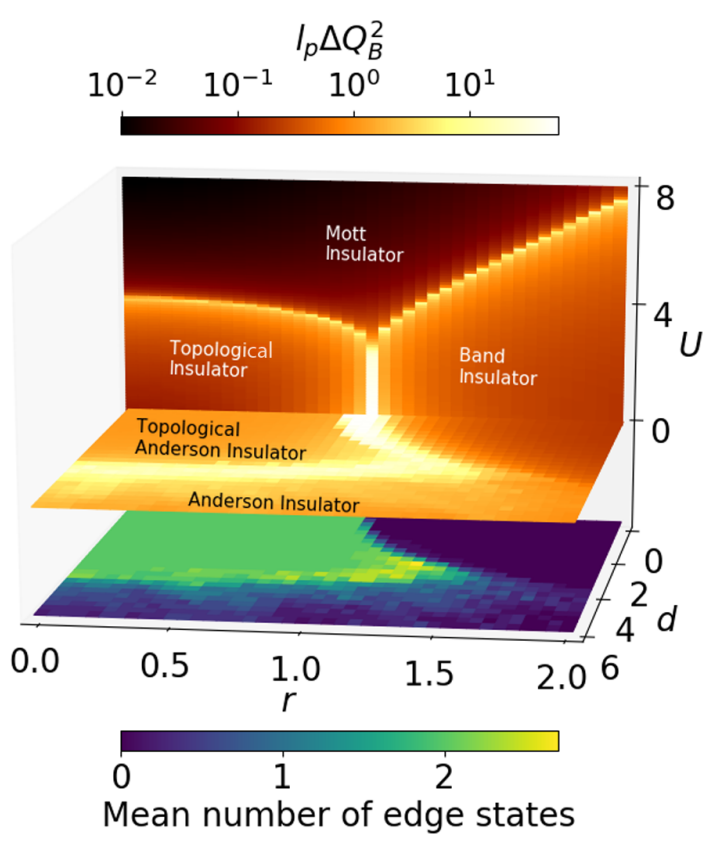

FIG. 1. Topological phase diagram characterized by the fluctuations $l_{p} \Delta Q_{B}^{2}$ (top) and by the number of zero-energy edge states (bottom) for the SSH model studied experimentally in [23]. $d$ is disorder strength and $U$ denotes nearest-neighbor Coulomb interaction. $r=t_{1} / t_{2}<1$ defines the topological region for $d=U=0$. Phase boundaries between topological and nontopological band, Anderson, and Mott insulators are all well characterized by strongly enhanced fluctuations [25]. 
of the gap size and reveals half-integer jumps at Weyl semimetal-like transitions [17-19]. Therefore, one expects strong fluctuations of $Q_{B}$ at a (topological) phase transition and it is quite surprising that these fluctuations have so far not drawn much attention [15].

We remedy this substantial oversight in this Letter and demonstrate that the fluctuations $\Delta Q_{B}^{2}=\left\langle\hat{Q}_{B}^{2}\right\rangle-\left\langle\hat{Q}_{B}\right\rangle^{2}$ of the boundary charge themselves are the key to addressing universal properties of (topological) phase transitions. We identify a "universal regime" $l_{p} \gg \xi_{g} \gg a$, where $l_{p} \Delta Q_{B}^{2}\left(\xi_{g}\right)$ is a universal function of $\xi_{g}$, i.e., independent of the microscopic details of the charge measurement probe, described by a macroscopic length scale $l_{p}$ on which the probe loses the contact to the sample (see below). Universality implies independence from high-energy properties, relevant on the scale of the lattice spacing $a$. In the regime close to the phase transition $\left(\xi_{g} \rightarrow \infty\right)$, we find that $l_{p} \Delta Q_{B}^{2}$ diverges in one and two dimensions, quite analog to divergent fluctuations in conventional phase transitions. Therefore, we suggest the fluctuations of $Q_{B}$ as a useful and measurable tool to probe the phase diagram of topological insulators. In Fig. 1 we begin by a compelling demonstration of the power of the suggested characterization, focusing on the prototypical Su-Schrieffer-Heeger (SSH) model $[21,22]$ at half filling, including hopping disorder (experimentally studied in Ref. [23]) and nearest-neighbor Coulomb interaction. Details of the model are postponed until Eq. (2), however, the general physics is dictated by the topological index $r$ being the ratio of the two hopping amplitudes in the SSH model. Without disorder and interaction, the topologically nontrivial phase transitions to a trivial one at $r=1$. Including disorder in the hoppings $d$, a topological Anderson insulator is stabilized even beyond $r=1$ for weak disorder, while for strong disorder, a trivial Anderson insulator is found. In the presence of strong enough Coulomb interaction $U$, a Mott insulator is established (for an interpretation of the Mott transition as a topological one, see Ref. [24]). All of the different phase boundaries between topological and nontopological band, Anderson, and Mott insulators are signaled by diverging boundary charge fluctuations $l_{p} \Delta Q_{B}^{2} \sim \xi_{g}$. Our approach thus unifies transitions between all of these different classes of single-particle and correlated insulators. In addition, below, we find that $l_{p} \Delta Q_{B}^{2}$ shows a universal scaling as a function of $E_{g}$ for a variety of models. We find a striking dependence on the dimensionality of the system, which we exemplify for a massive Dirac-like low-energy spectrum. We report a typical scaling with the inverse gap in one dimension, logarithmic scaling in two-dimensional systems, and a monotonic increase of the fluctuations to a finite value at zero-gap in three dimensions.

Model and boundary charge fluctuations.-We consider a generic and finite $D$-dimensional tight-binding model with a $D$-1-dimensional flat surface. In the direction perpendicular to the surface, we consider $N_{s}=L / a$ lattice

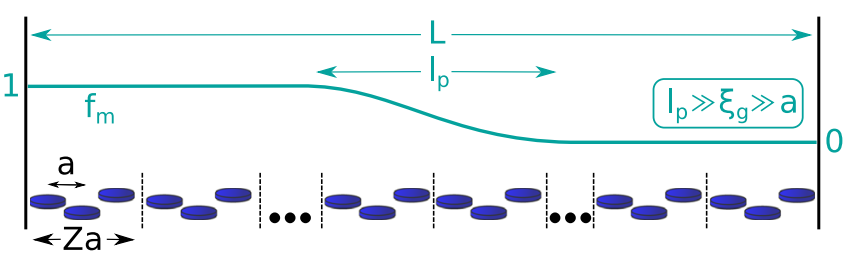

FIG. 2. Pictorial representation of the system (bottom) and the definition of the envelope of the charge probe $f_{m}$ (top). Here, $l_{p}$ defines the length on which the probe smoothly loses contact to the sample (region where envelope varies smoothly from 1 to 0 ). We also define the lattice spacing $a$ and the unit cell size $Z a$. Implicitly, we assume that the falloff of $f_{m}$ fits into the system size $L$ (the center of the falloff is irrelevant). The universal regime is defined by $l_{p} \gg \xi_{g}=v_{F} / E_{g} \gg a$.

sites labeled by $m=1, \ldots, N_{s}$ with open boundary conditions, where $L$ denotes the system size. We take an arbitrary extend and periodic boundary conditions in the remaining (transverse) directions. We then define an effective one-dimensional chain with two ends by absorbing the remaining directional degrees of freedom into a (large) multichannel character on each site, defining $N_{B}$ transverse channels labeled by $\sigma=1, \ldots, N_{B}$ (which can additionally include, e.g., spin or orbital degrees of freedom as well). The size of the unit cell of the effective onedimensional chain is denoted by $Z a$, and $x=m a$ defines the position of lattice site $m$; see Fig. 2 (bottom). We consider zero temperature and fixed particle number $N$ and concentrate on the low-energy limit, where the gap $E_{g}$ is assumed to be small compared to the bandwidth or, equivalently, $\xi_{g}=v_{F} / E_{g} \gg a$. Generalizations are discussed in the Supplemental Material [26]. We choose units $\hbar=e=1$.

The boundary charge is a macroscopic observable measured on scales much larger than the microscopic scale $\xi_{g} \gg a$. We describe the macroscopic average by an envelope function $f_{m}$ characteristic for a charge measurement probe, which falls off smoothly from unity to zero on the macroscopic length scale $l_{p} \gg \xi_{g}$, see Fig. 2 (top). The boundary charge operator at one end (determined by the envelope function $f_{m}$ falling off from that end) of the system is defined $[15,18]$ by $\hat{Q}_{B}=\sum_{m=1}^{N_{s}} f_{m}\left(\hat{\rho}_{m}-N / N_{s}\right)$, where $\hat{\rho}_{m}=\sum_{\sigma} a_{m \sigma}^{\dagger} a_{m \sigma}$ is the charge operator at site $m$ summed over all $N_{B}$ channels. The fluctuations $\Delta Q_{B}^{2}=$ $\left\langle\hat{Q}_{B}^{2}\right\rangle-\left\langle\hat{Q}_{B}\right\rangle^{2}$ can straightforwardly be expressed via the correlation function $C_{m m^{\prime}}=\left\langle\hat{\rho}_{m} \hat{\rho}_{m^{\prime}}\right\rangle-\left\langle\hat{\rho}_{m}\right\rangle\left\langle\hat{\rho}_{m^{\prime}}\right\rangle$ by exploiting the exact sum rule $\sum_{m^{\prime}=1}^{N_{s}} C_{m m^{\prime}}=0$. We obtain $\Delta Q_{B}^{2}=-\frac{1}{2} \sum_{m, m^{\prime}=1}^{N_{s}}\left(f_{m}-f_{m^{\prime}}\right)^{2} C_{m m^{\prime}}$. Employing that $C_{m m^{\prime}}$ decays exponentially for $\left|m-m^{\prime}\right| \gg \xi_{g}$, we find that the fluctuations are finite in the thermodynamic limit and the correlation function $C_{m m^{\prime}}$ can be replaced by the bulk correlation function $C_{m m^{\prime}}^{\text {bulk }} \equiv a^{2} C_{\text {bulk }}\left(x, x^{\prime}\right)$ as we have $l_{p} \gg \xi_{g}$. Expanding $f_{m}-f_{m^{\prime}}$ up to first order in $m-m^{\prime}$ 


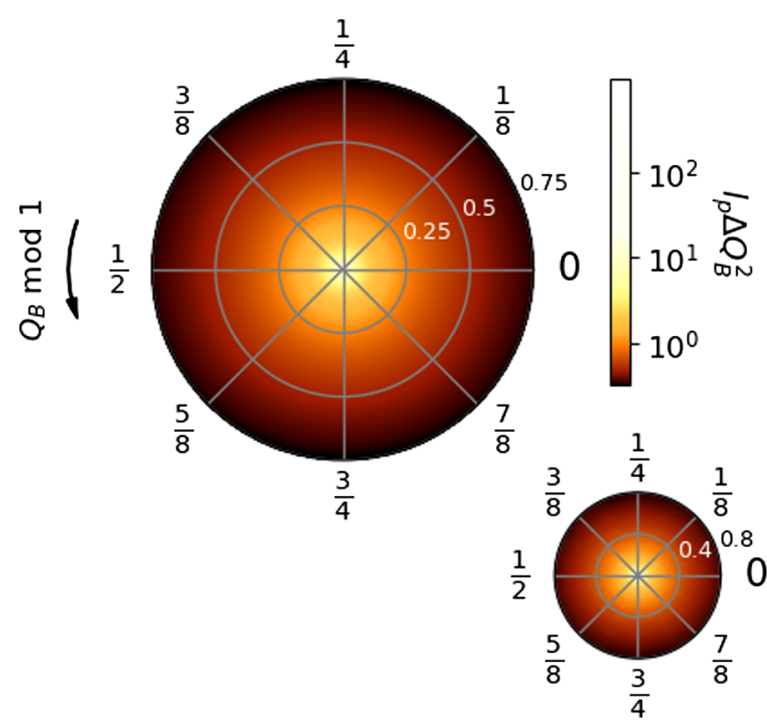

FIG. 3. Polar color plot of $l_{p} \Delta Q_{B}^{2}$ as a function of $Q_{B} \bmod (1)$ (polar component) and the gap $E_{g}$ (radial component) for model (2) with $Z=2, a=1, N / N_{s}=1 / 2, t=\left(t_{1}+t_{2}\right) / 2=1, d=0$, and $U=0$ (upper) or $U=0.5$ (lower). The data points are obtained by taking $t_{1 / 2}=t \pm \Delta_{0} / 2 \cos (\phi), v_{1 / 2}= \pm \Delta_{0} \sin (\phi)$, and varying the parameters $\Delta_{0}$ and $\phi$ in the intervals $\Delta_{0} \in$ $[0,0.375]$ and $\phi \in[0,2 \pi]$. For $U=0$ the analytic result $\left(N_{s}, l_{p} \rightarrow \infty\right)$ is used, while for $U=0.5$ we set $N_{s}=1000$, and $l_{p}=400$.

and averaging the correlation function over the unit cell [denoted by $\bar{C}_{\text {bulk }}\left(x, x^{\prime}\right)=\bar{C}_{\text {bulk }}\left(x-x^{\prime}\right)$ ], we find in the universal limit $l_{p} \gg \xi_{g} \gg a$ the result (see the Supplemental Material [26] for details)

$$
l_{p} \Delta Q_{B}^{2}=-\frac{1}{2} \int d x x^{2} \bar{C}_{\text {bulk }}(x)+O\left(\xi_{g}^{2} / l_{p}\right)
$$

where we defined $l_{p}^{-1}=\int d x f^{\prime}(x)^{2}$ with $f(m a) \equiv f_{m}$. Our result requires only the condition that $\bar{C}_{\text {bulk }}(x)$ decays exponentially for distances above the scale $\xi_{g}$. This is expected generically in the insulating regime due to the nearsightedness principle $[40,41]$. Here, $\xi_{g}$ should be considered as an upper limit for the decay length of $\bar{C}_{\text {bulk }}(x)$; in multichannel or interacting models, it is generically expected that $\bar{C}_{\text {bulk }}(x)$ consists of a linear combination of many exponentially decaying terms with different length scales $\xi_{\sigma} \lesssim \xi_{g}$ (see below the discussion of higher-dimensional systems).

We note that our central result (1) is independent of the scale $l_{p}$ (besides the condition $l_{p} \gg \xi_{g}$ ), offering a high degree of flexibility to measure and calculate the universality of boundary charge fluctuations; e.g., in cold atom systems, one can probe them either directly via the density profile or the correlation function [42]. Alternatively, for the special choice $f_{m}=1-m / N_{s}$ (where $l_{p}=L$ ), we get $\hat{Q}_{B}=-\hat{P} / L$ with $\hat{P}=a \sum_{m=1}^{N_{s}} m\left(\hat{\rho}_{m}-N / N_{s}\right)$ denoting the bulk polarization operator, the fluctuations of which are also discussed within localization theories [43]. Our result $l_{p} \Delta Q_{B}^{2}=\Delta P^{2} / L$ provides a "surface fluctuation theorem," connecting boundary and bulk fluctuations in a universal way. This can be viewed as the fluctuation-based analog of the celebrated surface charge theorem $[17,18,44,45]$.

Single-channel case.-A theory as general as the one outlined above can be put to the test in a plethora of applications. We start with the most simple single-channel case $N_{B}=1$ and nearest-neighbor hoppings, where numerically exact results in the clean or disordered case (by diagonalization of the single-particle problem), as well as in the presence of interactions, can be obtained with relative ease (in the interacting case by use of density matrix renormalization group approaches). We consider the following model:

$$
\begin{aligned}
H= & -\sum_{m=1}^{N_{s}-1}\left(t_{m}+w_{m}\right)\left(a_{m+1}^{\dagger} a_{m}+\text { H.c. }\right)+\sum_{m=1}^{N_{s}} v_{m} \rho_{m} \\
& +U \sum_{m=1}^{N_{s}}\left(\rho_{m}-1 / 2\right)\left(\rho_{m+1}-1 / 2\right),
\end{aligned}
$$

where $t_{m}=t_{m+Z}$ and $v_{m}=v_{m+Z}$ are periodically modulated nearest-neighbor hoppings and on-site potentials, respectively, $w_{m}$ describes bond disorder drawn from a uniform distribution $w_{m} \in\left[-d_{m} / 2, d_{m} / 2\right)$ with $d_{m}=d_{m+Z}$, and $U \geq 0$ is a nearest-neighbor repulsive interaction.

The phase diagram of this model in the SSH limit $[21,22]$ at half filling (choosing $Z=2$ and $v_{m}=0$ ) and its characterization in terms of the boundary charge fluctuations were already discussed above; see Fig. 1. Varying the interaction strength $U$ as well as hopping disorder $d=d_{1}=2 d_{2}$, gap closings indicated by strongly enhanced boundary charge fluctuations are found. In the $(r, d)$ plane at finite disorder and $U=0$, we show that our characterization in terms of the boundary charge fluctuations is perfectly consistent with the number of edge states, thus demonstrating perfect agreement with the theoretical [46] and experimental [23] findings. At finite $U$ the transition to the correlated Mott insulator is more involved and classification schemes are rare. The Mott insulator is characterized by a charge density wave instability due to umklapp processes [47], generating a staggered on-site potential. This potential breaks the chiral symmetry of the SSH model and leads to a nontopological phase. The boundary charge fluctuations provide a valuable tool to find also this transition line; compare Fig. 1. From exact solutions, one point of this transition line into the Mott insulator is known to be at $r=1$ $\left(t_{1}=t_{2}\right), U / t_{1}=2$, which is in perfect agreement with the boundary charge fluctuation characterization.

Whereas the boundary charge fluctuations depend strongly on the gap size, the boundary charge $Q_{B}$ itself is sensitive to the phase of the gap parameter in onedimensional, single-channel models [19]. This suggests the 


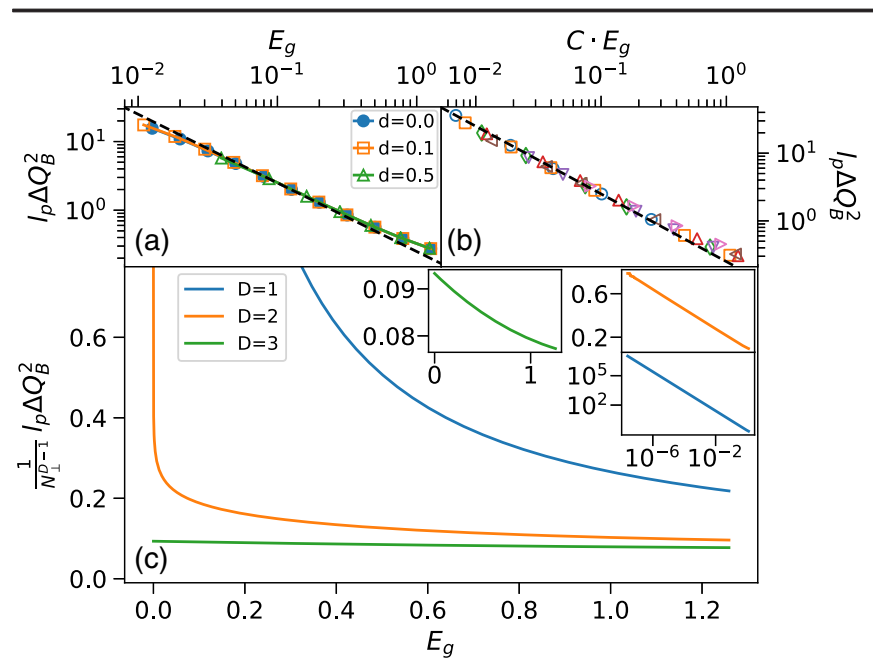

FIG. 4. (a),(b) Scaling of $l_{p} \Delta Q_{B}^{2}$ with the gap $E_{g}$ for model (2) on double-logarithmic scale for (a) $U=0$ and varying disorder strength $d$ (averaged over 20 disorder configurations) and (b) $d=0$ and varying Coulomb interaction strength $U$ (other parameters as in Fig. 1). The dashed line indicates $v_{F} /\left(8 E_{g}\right)=1 /\left(4 E_{g}\right)$. (b) $U=(0.0,0.4,0.8,1.2,1.6,2.0,2.4)$ are given by (blue, orange, green, red, purple, brown, pink) symbols. The nonuniversal prefactors $C(U)$ for the collapse are given in the Supplemental Material [26]. In (a) and (b) we choose $N_{s}=1000$ and $l_{p}=400$. (c) Scaling of $l_{p} \Delta Q_{B}^{2} / N_{\perp}^{D-1}$ from the analytic result $\left(N_{s}, l_{p} \rightarrow \infty\right)$ with the gap $E_{g}=2\left|t_{1}-t_{2}\right|$ for various dimensions $D$ for the SSH model $(D=1)$ and for the higher-dimensional models discussed in the main text $(D=2,3)$ with $t=\left(t_{1}+t_{2}\right) / 2=t_{y}=t_{z}=1$. Insets: the same results on different scales.

polar plot of Fig. 3, where we show the fluctuations $l_{p} \Delta Q_{B}^{2}$ in dependence of the gap $E_{g}$ (radial component) and the boundary charge $Q_{B} \bmod (1)$ (polar component) for the model of Eq. (2) with $Z=2$ and $d=0$, both for $U=0$ and finite $U$, choosing a variety of parameters to define the staggered on-site potentials $v_{1}=-v_{2}$ and hoppings $t_{1 / 2}$. This corresponds to the noninteracting and interacting Rice-Mele model $[20,48]$. The radially symmetric value of $l_{p} \Delta Q_{B}^{2}$ indicates that the fluctuations depend only on the gap's absolute value, but not on $Q_{B}$, and that they strongly enhance at the center $E_{g} \rightarrow 0$. We expect this feature to be generic for one-dimensional, single-channel models in the low-energy regime.

For the noninteracting and clean Rice-Mele model $Z=2$, we find analytically the exact result (see the Supplemental Material for details [26]) $l_{p} \Delta Q_{B}^{2}=$ $a\left(t_{1}^{2}+t_{2}^{2}\right) /\left(4 E_{g} \sqrt{E_{g}^{2} / 4+4 t_{1} t_{2}}\right)$, with the gap $E_{g}=$ $2 \sqrt{v^{2}+\left(t_{1}-t_{2}\right)^{2}}$ and $v=v_{1}=-v_{2}$. In the vicinity of phase transitions $E_{g} \ll t=\left(t_{1}+t_{2}\right) / 2$, we obtain the universal scaling

$$
l_{p} \Delta Q_{B}^{2}\left(E_{g} \stackrel{E_{g} \ll t}{\rightarrow} \frac{v_{F}}{8 E_{g}}=\frac{\xi_{g}}{8},\right.
$$

where $v_{F}=2 t a$ denotes the Fermi velocity. For arbitrary value of $Z$ and generic modulations of the nearestneighbor hoppings and the on-site potentials, we confirm this universal scaling for the chemical potential located in any gap. We obtain this result by using the exact eigenstates of a low-energy massive Dirac model in $1+$ 1 dimensions, as proposed in Ref. [19] (see the Supplemental Material [26] for details). Furthermore, we find that $\xi_{g}=v_{F} / E_{g}$ is the exponential decay length of the correlation function $\bar{C}_{\text {bulk }}(x)$. For more exotic models that cannot be described by a Dirac model in the low-energy regime, we show in the Supplemental Material [26] that also other scalings are, in principle, possible. For the noninteracting and clean SSH model, we numerically confirm the scaling $l_{p} \Delta Q_{B}^{2}=v_{F} /\left(8 E_{g}\right)$ in Fig. 4(a) and find that it holds up to surprisingly large gaps even beyond the applicability range of the low-energy theory. This relation holds also in the presence of disorder, at least for not too strong disorder $d \lesssim 2-3$, where a Born approximation [49] can be used to define a renormalized gap $E_{g}=2\left|\bar{t}_{1}-\bar{t}_{2}\right|$, with $\bar{t}_{1}=t_{1}-\theta\left(t_{1}-t_{2}\right) d_{1}^{2} /\left(12 t_{1}\right)$ and $\bar{t}_{2}=t_{2}-\theta\left(t_{2}-t_{1}\right) d_{2}^{2} /\left(12 t_{2}\right)$ denoting renormalized hoppings (see the Supplemental Material for details [26]). In Fig. 4(b) we show the scaling for different $U$ and $d=0$ and find that they collapse to the universal $\sim 1 / E_{g}$ if one allows for a $U$-dependent nonuniversal prefactor [26]. In this case, the gap is significantly increased by interactions $[19,20,50-52]$.

Two- and three-dimensional systems.-To analyze the scaling in two and three dimensions $D=2,3$ for noninteracting and clean systems, we use translational invariance in the transverse direction and consider $N_{B}=N_{\perp}^{D}$ transverse quasimomenta $\underline{k}_{\perp}$ as a channel index. The fluctuations of $Q_{B}$ can then be calculated as an independent sum $\Delta Q_{B}^{2}=\sum_{\underline{k}_{\perp}} \Delta Q_{B}^{2}\left(\underline{k}_{\perp}\right)$, with $k_{\perp}=k_{y}$ for $D=2$ and $\underline{k}_{\perp}=\left(k_{y}, k_{z}\right)$ for $D=3$. For each fixed $k_{\perp}$, we consider an effective one-dimensional, singlechannel system and get from Eq. (3) in the low-energy regime $l_{p} \Delta Q_{B}^{2}\left(\underline{k}_{\perp}\right)=v_{F}\left(\underline{k}_{\perp}\right) /\left[8 E_{g}\left(\underline{k}_{\perp}\right)\right]$, corresponding via Eq. (1) to an independent term of $\bar{C}_{\text {bulk }}(x)$ decaying on length scale $\xi\left(\underline{k}_{\perp}\right)=v_{F}\left(\underline{k}_{\perp}\right) / E_{g}\left(\underline{k}_{\perp}\right) \leq \xi_{g}$. The momentum dependence of the effective gap $E_{g}\left(\underline{k}_{\perp}\right)$ can be estimated for a typical massive Dirac-like spectrum in $D+1$ dimensions: $E_{g}\left(\underline{k}_{\perp}\right) \approx 2 \sqrt{\bar{v}_{F}^{2} k_{\perp}^{2}+E_{g}^{2} / 4}$, where $E_{g}$ denotes the spectral gap, and we have neglected the weak momentum dependence of $v_{F}\left(\underline{k}_{\perp}\right) \approx \bar{v}_{F}$. In the thermodynamic limit $\sum_{\underline{k}_{\perp}} \rightarrow\left(N_{\perp} a /(2 \pi)\right)^{D-1} \int_{-\pi / a}^{\pi / a} d^{D-1} k_{\perp}$, we can estimate the scaling of the fluctuations. In two dimensions, we obtain a logarithmic scaling $N_{\perp}^{-1} l_{p} \Delta Q_{B}^{2} \sim$ $\left(\bar{v}_{F} / W\right) \ln \left(W / E_{g}\right) \sim a \ln \left(\xi_{g} / a\right)$, where $W$ defines a highenergy cutoff scale for $\left|\bar{v}_{F} k_{y}\right|$. In contrast, for three dimensions, we obtain a monotonic increase for the 
fluctuations with decreasing gap but a finite value in the zero-gap limit.

Systems illustrating this generic behavior can be realized, e.g., in cold atom systems [53]. As an example, we consider a SSH model in $x$ direction (with alternating hoppings $t_{1,2}$ ), constant nearest-neighbor hoppings in transverse direction (denoted by $t_{y, z}$ ), and a homogeneous magnetic field of size $B$ in $z$ direction (for $D=2$ ) or in the $y$ and $z$ direction (for $D=3$ ). For the simplest case in which the magnetic length is given by $\lambda_{B}=2 a$, we obtain in the Landau gauge an effective one-dimensional RiceMele model with $E_{g}\left(\underline{k}_{\perp}\right)=2 \sqrt{v\left(\underline{k}_{\perp}\right)^{2}+\left(t_{1}-t_{2}\right)^{2}}$, where $v\left(\underline{k}_{\perp}\right)=2 t_{y} \cos \left(k_{y} a\right)$ in $D=2$ or $v\left(\underline{k}_{\perp}\right)=2 t_{y} \cos \left(k_{y} a\right)+$ $2 t_{z} \cos \left(k_{z} a\right)$ in $D=3$ (see the Supplemental Material [26] for details). Using the exact result for the Rice-Mele model to calculate $l_{p} \Delta Q_{B}^{2}\left(\underline{k}_{\perp}\right)$, one can perform the integral over $\underline{k}_{\perp}$ and finds for the fluctuations as a function of the gap $E_{g}=2\left|t_{1}-t_{2}\right|$ the result shown in Fig. 4(c). The logarithmic scaling in $D=2$ is perfectly preserved even for large gaps, suggesting the boundary charge fluctuations to be also useful as an indicator for phase transitions in two dimensions. We emphasize that, for $D=3$, we observe only a weak increase of the fluctuations with a finite value at zero gap. Therefore, for three dimensions, the fluctuations are only a weak indicator for the transition. Although this limits the universally diverging behavior to one- and two-dimensional systems, future studies should address whether also the nondivergent enhancement carries valuable information of the type of transition passed. In addition, it will be of interest to study how generic the proposed decoupling in transverse modes will persist in higher-dimensional interacting and disordered systems.

Conclusion.-We have established the boundary charge and its fluctuations as a measurable tool to probe topological properties of insulators. Whereas the boundary charge takes the role of a phase and jumps by $e / 2$ at a topological phase transition [19], the complementary fluctuations are strongly enhanced in one and two dimensions and reveal a universal scaling as a function of the gap size. In contrast to the number of topological edge states, which is controlled by nonfluctuating topological indices, we found that the universal scaling properties of the fluctuations do not depend on whether a topological index changes at the transition, but rely exclusively on the characteristic band structure of insulators with topological properties. Importantly, this characterization scheme can be applied to band, Anderson, and Mott insulators alike. An intriguing avenue of future research concerns the question whether the characterization proposed here is also useful in the context of topological superconductors, for which simple models do not fulfill charge conservation.

We thank V. Meden and D. Schuricht for fruitful discussions. This work was supported by the Deutsche Forschungsgemeinschaft via RTG 1995, the Swiss National
Science Foundation (SNSF) and NCCR QSIT, and by the Deutsche Forschungsgemeinschaft (DFG, German Research Foundation) under Germany's Excellence Strategy-Cluster of Excellence Matter and Light for Quantum Computing (ML4Q) EXC 2004/1390534769. We acknowledge support from the Max Planck-New York City Center for Nonequilibrium Quantum Phenomena. Simulations were performed with computing resources granted by RWTH Aachen University under project thes0753. Funding was received from the European Union's Horizon 2020 research and innovation program (ERC Starting Grant No. 757725).

C.S.W., K.P., and M.P. contributed equally to this work.

"Dante.Kennes@rwth-aachen.de

[1] B. A. Volkov and O. A. Pankratov, JETP Lett. 42, 178 (1985).

[2] O. Pankratov, S. Pakhomov, and B. Volkov, Solid State Commun. 61, 93 (1987).

[3] C. L. Kane and E. J. Mele, Phys. Rev. Lett. 95, 146802 (2005).

[4] B. A. Bernevig, T. L. Hughes, and S.-C. Zhang, Science 314, 1757 (2006).

[5] L. Fu, C. L. Kane, and E. J. Mele, Phys. Rev. Lett. 98, 106803 (2007).

[6] M. König, S. Wiedmann, C. Brüne, A. Roth, H. Buhmann, L. W. Molenkamp, X.-L. Qi, and S.-C. Zhang, Science 318, 766 (2007).

[7] D. Hsieh, D. Qian, L. Wray, Y. Xia, Y. S. Hor, R. J. Cava, and M.Z. Hasan, Nature (London) 452, 970 (2008).

[8] M.Z. Hasan and C. L. Kane, Rev. Mod. Phys. 82, 3045 (2010).

[9] X.-L. Qi and S.-C. Zhang, Rev. Mod. Phys. 83, 1057 (2011).

[10] B. A. Bernevig and T. L. Hughes, Topological Insulators and Topological Superconductors, student ed. (Princeton University Press, Princeton, NJ, 2013).

[11] G. Tkachov, Topological Insulators: The Physics of Spin Helicity in Quantum Transport (Pan Stanford, Singapore, 2015).

[12] J. K. Asbóth, L. Oroszlány, and A. Pályi, A Short Course on Topological Insulators, Lecture Notes in Physics Book Series (Springer, Cham, 2016).

[13] W. Kohn, Phys. Rev. 133, A171 (1964).

[14] R. Resta, J. Phys. Condens. Matter 14, R625 (2002).

[15] J.-H. Park, G. Yang, J. Klinovaja, P. Stano, and D. Loss, Phys. Rev. B 94, 075416 (2016).

[16] M. Thakurathi, J. Klinovaja, and D. Loss, Phys. Rev. B 98, 245404 (2018).

[17] M. Pletyukhov, D. M. Kennes, J. Klinovaja, D. Loss, and H. Schoeller, Phys. Rev. B 101, 161106(R) (2020).

[18] M. Pletyukhov, D. M. Kennes, J. Klinovaja, D. Loss, and H. Schoeller, Phys. Rev. B 101, 165304 (2020).

[19] M. Pletyukhov, D. M. Kennes, K. Piasotski, J. Klinovaja, D. Loss, and H. Schoeller, Phys. Rev. Research 2, 033345 (2020). 
[20] Y.-T. Lin, D. M. Kennes, M. Pletyukhov, C. S. Weber, H. Schoeller, and V. Meden, Phys. Rev. B 102, 085122 (2020).

[21] W. P. Su, J. R. Schrieffer, and A. J. Heeger, Phys. Rev. Lett. 42, 1698 (1979).

[22] W. P. Su, J. R. Schrieffer, and A. J. Heeger, Phys. Rev. B 22 , 2099 (1980).

[23] E. Meier, F. An, A. Dauphin, M. Maffei, P. Massignan, T. Hughes, and B. Gadway, Science 362, 929 (2018).

[24] S. Sen, P. J. Wong, and A. K. Mitchell, Phys. Rev. B 102, 081110(R) (2020).

[25] The other parameters are $N_{s}=400, l_{p}=200$ for $d \neq 0$ and $N_{s}, l_{p} \rightarrow \infty$ for $d=0$ (see Supplemental Material [26] for details on how the infinite system size limit can be taken numerically), $t_{2}=1, t_{1}=r, a=1$, and we work at half filling. The results are averaged over 20 disorder configurations. For the details of the model, see Eq. (2).

[26] See Supplemental Material at http://link.aps.org/ supplemental/10.1103/PhysRevLett.126.016803 for the $C$ 's used in Fig. 4 and details about the calculations in the noninteracting cases considered, as well as a discussion of finite temperature effects, which includes Refs. [27-39].

[27] S. Kivelson and J. R. Schrieffer, Phys. Rev. B 25, 6447 (1982).

[28] R. Rajaraman and J. Bell, Phys. Lett. 116B, 151 (1982).

[29] S. Kivelson, Phys. Rev. B 26, 4269 (1982).

[30] J. Bell and R. Rajaraman, Nucl. Phys. B220, 1 (1983).

[31] Y. Frishman and B. Horovitz, Phys. Rev. B 27, 2565 (1983).

[32] R. Jackiw, A. Kerman, I. Klebanov, and G. Semenoff, Nucl. Phys. B225, 233 (1983).

[33] J. J. Rehr and W. Kohn, Phys. Rev. B 10, 448 (1974).

[34] C. Kallin and B. I. Halperin, Phys. Rev. B 29, 2175 (1984).

[35] L. He and D. Vanderbilt, Phys. Rev. Lett. 86, 5341 (2001).

[36] C. S. Weber et al. (to be published).

[37] D. J. Thouless, M. Kohmoto, M. P. Nightingale, and M. den Nijs, Phys. Rev. Lett. 49, 405 (1982).
[38] N. Hine and M. Foulkes, J. Phys. Condens. Matter 19, 506212 (2007).

[39] H. Min and A. H. MacDonald, Prog. Theor. Phys. Suppl. 176, 227 (2008).

[40] W. Kohn, Phys. Rev. Lett. 76, 3168 (1996).

[41] E. Prodan and W. Kohn, Proc. Natl. Acad. Sci. U.S.A. 102, 11635 (2005).

[42] I. Bloch, J. Dalibard, and W. Zwerger, Rev. Mod. Phys. 80, 885 (2008).

[43] C. Sgiarovello, M. Peressi, and R. Resta, Phys. Rev. B 64, 115202 (2001).

[44] D. Vanderbilt and R. D. King-Smith, Phys. Rev. B 48, 4442 (1993).

[45] D. Vanderbilt, Berry Phases in Electronic Structure Theory: Electric Polarization, Orbital Magnetization and Topological Insulators (Cambridge University Press, Cambridge, England, 2018).

[46] I. Mondragon-Shem, T. L. Hughes, J. Song, and E. Prodan, Phys. Rev. Lett. 113, 046802 (2014).

[47] T. Giamarchi, Quantum Physics in One Dimension, International Series of Monographs on Physics (Oxford University Press, Oxford, 2003).

[48] M. J. Rice and E. J. Mele, Phys. Rev. Lett. 49, 1455 (1982).

[49] C. W. Groth, M. Wimmer, A. R. Akhmerov, J. Tworzydło, and C. W. J. Beenakker, Phys. Rev. Lett. 103, 196805 (2009).

[50] S. Kivelson, H. B. Thacker, and W.-K. Wu, Phys. Rev. B 31, 3785(R) (1985).

[51] B. Horovitz and J. Sólyom, Phys. Rev. B 32, 2681 (1985).

[52] S. Gangadharaiah, L. Trifunovic, and D. Loss, Phys. Rev. Lett. 108, 136803 (2012).

[53] N. R. Cooper, J. Dalibard, and I. B. Spielman, Rev. Mod. Phys. 91, 015005 (2019). 